\title{
The Many Faces of the Gas in Centaurus A (NGC 5128)
}

\author{
Raffaella Morganti \\ Netherlands Institute for Radio Astronomy, Postbus 2, 7990 AA, Dwingeloo, \\ and Kapteyn Astronomical Institute, University of Groningen, P.O. Box 800, \\ 9700 AV Groningen, The Netherlands. Email: morganti@astron.nl
}

Received 2009 October 15, accepted 2010 February 28

\begin{abstract}
Centaurus A (NGC 5128) is ideal for investigating the characteristics and role of gas in an earlytype galaxy in the presence of a radio-loud active nucleus. The different phases of the gas - hot (X-ray), warm (ionized), and cold (H I and molecular) — are all detected in this object and can be studied at very high spatial resolution, due to its proximity. This richness makes Centaurus A truly unique. Spatially, these gas structures span from the pc to the tens of kpc scale. Thus, they allow us to trace very different phenomena, from the formation and evolution of the host galaxy, to the interplay between nuclear activity and interstellar medium and the feeding mechanism of the central black hole. A lot of work has been done to study and understand the characteristics of the gas in this complex object, and the present paper summarizes what has been achieved so far.
\end{abstract}

Keywords: galaxies: individual (Centaurus A/NGC 5128) — galaxies: ISM — galaxies: active

\section{Introduction}

In recent years, there has been a change of paradigm in our view of early-type galaxies. Recent detailed observations of these objects in different wavebands have confirmed and emphasized the picture - already suggested in earlier studies (see, e.g., Kormendy \& Bender 1996; Faber et al. 1997 and references therein) - that the structure, dynamics, stellar populations, and nuclear activity of early-type galaxies is complex and, therefore, early-type galaxies do not constitute a uniform class. In addition, it has become clear that gas is an important ingredient, even in these objects, and an element that cannot be neglected (see, e.g., Bender, Burstein \& Faber 1992; Cappellari et al. 2007 and references therein). Recent observations, deeper than available before, have shown that early-type galaxies do have a rich, complex, and multiphase interstellar medium (ISM) which contains hot (X-ray), warm (ionized) and cold ( $\mathrm{H}$ I and molecular) components. We know that early-type galaxies can contain large quantities - up to at least several $10^{7} \mathrm{M}_{\odot}$ - of hot gas (see Mathews \& Brigenti 2003 for a review). However, for the remaining phases of the gas and in particular cold gas, early-type galaxies used to be perceived as gas poor. Although, indeed, they typically have less cold gas than spiral galaxies, it is now clear that cold gas is detected in many early-type galaxies, provided that deep observations are available (see, e.g., Morganti et al. 2006; Sarzi et al. 2006; Combes, Young \& Bureau 2007). The variety of characteristics of their gaseous structures can be considered as a signature of the continuing assembly of these objects. The relevance of gas in the formation and evolution of early-type galaxies is also suggested by theoretical work that indicates that dissipative mergers and accretion events are needed to explain the dynamical structure of the more disky early-type galaxies, in particular (see, e.g., Naab \& Burkert 2003; Bournaud, Jog \& Combes 2005; Hopkins et al. 2009).

Inside this revised picture of early-type galaxies, Centaurus A (Cen A) represents a nice example of the complexity described above. Cen A (NGC 5128) ${ }^{1}$ is an early-type galaxy (see Harris et al. 2010) with a minor axis dust lane and a major axis stellar component, therefore showing a strong misalignment between stars and gas, relatively common in early-type galaxies. Cen A has the exciting property of showing the presence of different phases of the gas with structures spread across a wide range of spatial scales, going from the sub-pc scale up to tens of kpc. Thanks to its proximity, Cen A offers the possibility of exploring the structure and kinematics of the gas with very high spatial resolution. This, combined with the fact that it is an early-type galaxy hosting a radioloud active galactic nucleus (AGN), makes Cen A a truly unique object.

A lot of work has been done to study and understand the characteristics of the gas in Cen A. The gas has been used to trace different phenomena and help in understanding the characteristics of this galaxy. In

\footnotetext{
${ }^{1}$ To be correct, I should be using NGC 5128 when discussing the properties of the optical galaxy, however for convenience I will be using Cen A, which is in fact the name of the radio source, throughout this paper.
} 
particular - and more related to the case of an active nucleus like Cen A - the study of the gas allows us to:

- probe the radial mass distribution and the shape of early-type galaxies, which also provides information on conditions (and timescales) during their formation;

- trace signatures of mergers/interactions or recent accretions, again giving information about the formation history of the galaxy;

- understand the nuclear structure, in particular through the study of gas kinematics, which provides information on the fuelling mechanism, a still open question especially for relatively low-power radio galaxies like Cen $\mathrm{A}$; and

- trace the presence of outflows and the effects of AGNrelated feedback.

It is worth noting that Cen A has clear evidence (dust lane, shells, etc.) that the gas has been brought in through the accretion of a small gas-rich galaxy. This is not always clear in other early-type galaxies. In fact, although mergers can provide a good description of many characteristics of early-type galaxies, it is nevertheless important to keep in mind that gas can also be acquired in other ways. Particularly interesting is the cold accretion, the slow but long-lasting infall of primordial gas (see, e.g., Keres et al. 2005; Dekel et al. 2009). Thus, in the case of Cen A we can really study the effects of merger in shaping the characteristics of the galaxy, including the presence of a radio-loud AGN.

This paper attempts to give an overview of the many faces of the gas in Cen A, although, given the extremely extensive literature available on this subject, it is impossible for it to be a complete review. The rest of the paper describes the gaseous structures in Cen A starting from the large scale and then zooming in toward the inner, nuclear regions. The paper first describes the gas observed up to $\sim 20 \mathrm{kpc}$ from the centre. After that, it will move to the gas observed along the dust lane (up to $\sim 6 \mathrm{kpc}$ from the centre). Finally, it will explore the sub-kpc and nuclear $(<30 \mathrm{pc})$ regions to investigate possible effects of the radio jet and of the black hole $(\mathrm{BH})$. Furthermore, the paper looks at the possible effects of the interaction between the large scale radio emission and the gas. For a more general review of Cen $\mathrm{A} / \mathrm{NGC} 5128$ see Israel (1998).

\section{Gas in Cen A: Large-Scale Structures}

At large distances from the centre, the ISM of Cen A shows a complex and intriguing structure. Most of the action happens in the north-east side of the galaxy, the side where the most complex part of the radio emission is also observed (the so-called northern middle lobe and the outer lobe). Whether there is a connection between these two facts - e.g., due to the presence of a particularly dense external medium at this location (see the results on the hot gas at the end of this section) - is not yet clear. The location of the various gas structures is illustrated in Figure 1.

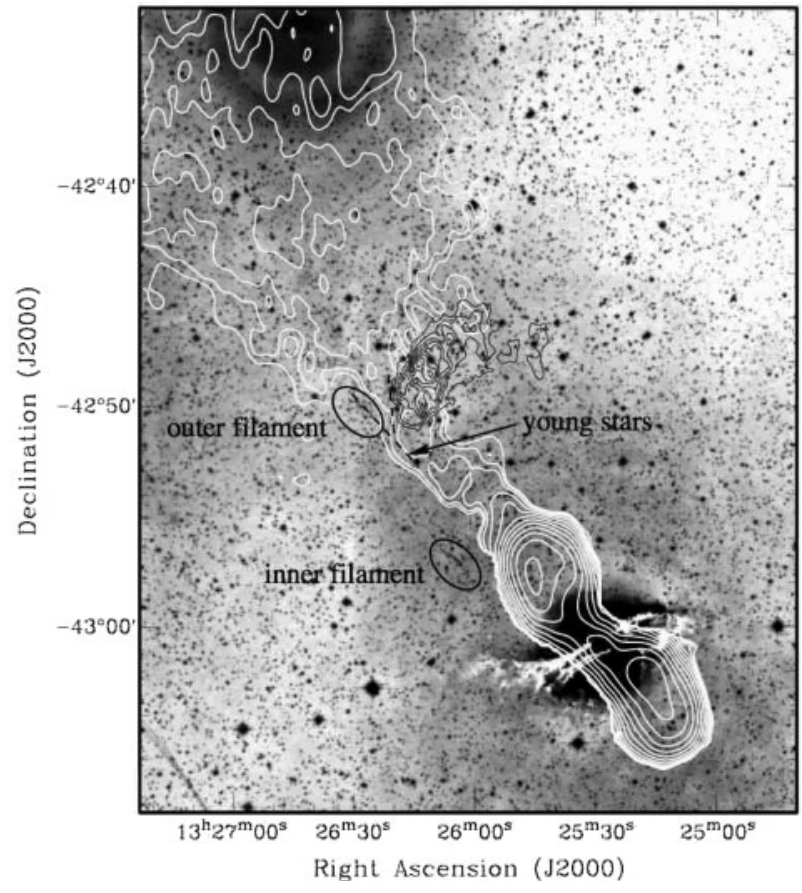

Figure 1 Overlay showing the various components described in the text. The optical image (kindly provided by D. Malin) shows the well-known dust lane of Cen $\mathrm{A}$ and the faint diffuse optical emission that extends to large distances from the centre. The white contours denote the radio continuum emission (after Morganti et al. 1999a) showing the bright inner radio lobes and the large-scale jet that connects these lobes to the so-called northern middle lobe in the topleft of the figure. The black contours denote the $\mathrm{H}$ i cloud discussed in this paper (see also Figure 3). The locations of the inner and outer filaments of highly ionized gas are indicated, as well as the location of young stars.

Highly ionized filaments of gas have been observed in this region, extending up to at least $20 \mathrm{kpc}$ from the nucleus to the northern side of the galaxy. The extended filaments were discovered by Blanco et al. (1975): the inner filament located at a projected distance from the centre of $7 \mathrm{kpc}$ and extended $\sim 2 \mathrm{kpc}$ and the outer filament located at a projected distance of $16 \mathrm{kpc}$. In both structures (but in particular in the inner filament) the gas shows very high ionization. An example of optical spectrum is shown in Figure 2. More closer to the centre, Dufour \& van den Bergh (1978) pointed out the presence of an inner optical jet made of compact knots and diffuse filaments but this structure was not confirmed by narrow band images (Morganti et al. 1991). Interestingly, no similar filaments have been found in the southern part of the galaxy.

The emission line spectra of the filaments are very similar in character to those of the spatially extended nebulosities in more distant radio galaxies and radio quasars. From the analysis of these lines, Morganti et al. (1991) concluded that the filaments are photoionized by the radiation field of a nuclear continuum source which is hidden from our direct view either by obscuration or by intrinsic anisotropy (or both). This hypothesis is supported by the ionization gradient (i.e. higher ionization 


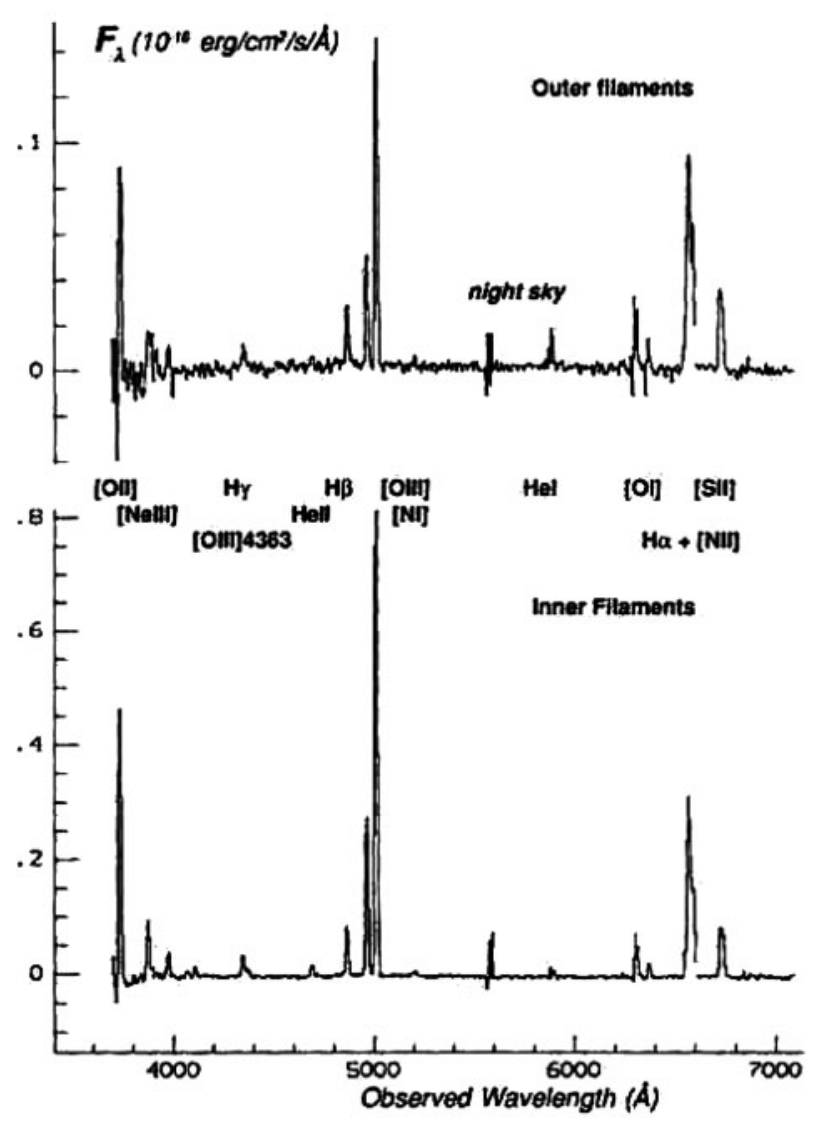

Figure 2 An example of the inner and outer filament spectra taken from Morganti et al. (1991). The principal emission lines are labelled.

on the side closer to the nucleus) observed in the inner filament (Morganti et al. 1992). The energy required to photoionize the gas would be consistent with Cen A having a beam power similar to that of BL Lac. The similarity between Cen A and BL Lac is also confirmed by the near-infrared (IR) study and the study of the spectral energy distribution by Marconi et al. (2000).

However, in addition to the high excitation, the gas in the filaments shows velocity variations of 100 $200 \mathrm{~km} \mathrm{~s}^{-1}$ happening over regions of only a few hundred pc. This complex kinematics cannot be accounted for by radiation pressure. Particle beam acceleration seems to be necessary, thus suggesting the possibility (explored by Sutherland, Bicknell \& Dopita 1993) that the ionization of the filaments is actually connected to shocks produced by the interaction of the radio jet with the ISM. However, at least the inner filament does not appear to (by looking at the radio image) experience a direct interaction with the radio jet that would produce fast enough shocks and, consequently, the high ionization observed (see Morganti et al. 1999). Thus, it is more likely that a combination of both mechanisms provides the observed characteristics. In this respect, the region where the outer filaments are located is particularly interesting. The radio emission (in the form of a collimated structure, see Morganti et al. 1999) appears, in projection, to pass next to the outer filaments of highly ionized gas and next to a large cloud/ tail of neutral gas (Schiminovich et al. 1994); see Figure 1 for an overview of the location of these structures. The location of the H i next to the filaments of ionized gas suggests that a narrow radiation beam could be the origin of the ionization. However, higher velocity resolution $\mathrm{H}_{\mathrm{I}}$ observations (Oosterloo \& Morganti 2005) have revealed that, apart from the smooth velocity gradient corresponding to the overall rotation of the $\mathrm{H}$ I cloud/tail around Cen A, $\mathrm{HI}$ with anomalous velocities of about $100 \mathrm{~km} \mathrm{~s}^{-1}$ is present at the southern tip of this cloud. This has been interpreted as evidence for an ongoing interaction between the radio plasma and the $\mathrm{H}_{\mathrm{i}}$ cloud.

All this is even more interesting considering that groups of young stars have been found in the same region. The estimated ages of these stars are about $15 \mathrm{Myr}$ (see Mould et al. 2000; Rejkuba et al. 2002). Recent GALEX data (Neff, Schiminovich \& Martin 2003 and these Proceedings) also indicate the presence of ultraviolet emission that appears to be related not only to the optical filaments, but also to the chain of young stars. Graham (1998) suggested for the first time the possibility discussed later by many other authors - that the interaction between the radio jet and the ISM could also trigger star formation. The signature of an on-going interaction seen in the kinematics of the $\mathrm{H}$ i appears to further support this idea. Oosterloo \& Morganti (2005) have suggested that gas stripped from the $\mathrm{H}$ i cloud would give rise to both the large filament of ionized gas and the star formation regions that are found downstream from the location of the interaction. Given the amount of $\mathrm{H}$ I with anomalous kinematics and the current star formation rate, the efficiency of jet-induced star formation is at most of the order of a percent.

As a final remark on the ionized gas found in the filaments, it is worth mentioning that regions of ionized gas at large distances from the nucleus are observed in many other radio sources (Baum et al. 1988). However, some cases appears particularly similar to Cen A. Examples of these objects are PKS 2152-69 (Fosbury et al. 1998) and IC 2497 (so-called 'Hanny's voorwerp'; Józsa et al. 2009).

As mentioned above, $\mathrm{H}_{\mathrm{I}}$ has been detected extending up to $\sim 15 \mathrm{kpc}$ from the centre (Schiminovich et al. 1994; Oosterloo \& Morganti 2005) distributed in a structure that appears consistent with a partially rotating ring with the same sense of rotation as the main body of the galaxy. It is intriguing that some of the $\mathrm{H}_{\mathrm{I}}$ is roughly associated with optical shells (on the northern side of the galaxy; Schiminovich et al. 1994), also see Figure 3. The presence of $\mathrm{H}$ in the shells is not expected: the dynamics of the gas and stellar component is expected to decouple during a merger event and, due to dissipation, the gas rapidly concentrates in the nucleus and, unlike stars, does not form shell. A possible explanation is that the structure is the result of the interaction between the $\mathrm{H}$ I and the X-ray halo. Indeed, Cen A has an extended halo with as much as $50 \%$ of the X-ray coming from a hot corona (Forman, Jones \& Tucker 1985). The corona extends out to the $\mathrm{H}_{\mathrm{I}}$ 


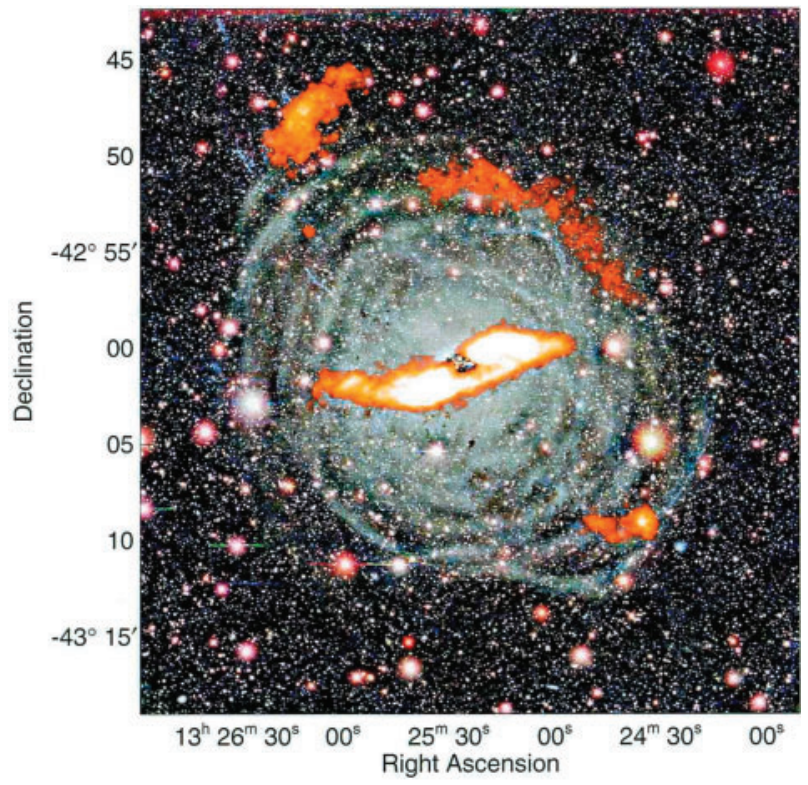

Figure $3 \mathrm{HI}$ emission obtained from the ATCA observations (orange) superimposed on an optical BVR image obtained after unsharp masking and adaptive histogram equalization by Peng et al. (2002). The optical image emphasizes the low-contrast features and in particular the complex set of shells and the faint dust extensions. This overlay was kindly provided by $\mathrm{T}$. Oosterloo.

associated with the shells (see the overlay in figure 5 in Schiminovich et al. 1994), suggesting the possibility of an interaction between the hot and the cold gas.

It is interesting to note that molecular gas has also been found at the location of the shells (Charmandaris, Combes \& van der Hulst, see Figure 2). Surprisingly, the location of the molecular gas is offset compared to the location where the young stars are found and no young stars seem to be present in coincidence with the CO. The ratio $\mathrm{M}\left(\mathrm{H}_{\mathrm{I}}\right) / \mathrm{MH}_{2}$ is, in these regions, close to unity and similar to what found in the central regions.

Charmandaris et al. (2000) suggest that the molecular gas could be originating from the merger and, while the diffuse atomic gas at these distances is expected to lose its energy and fall toward the centre, much denser $\left(10^{3} \mathrm{~cm}^{-3}\right)$ molecular clouds may be expected to survive at these locations. On the other hand, the presence of $\mathrm{CO}$ could help explain the atomic neutral hydrogen at the location of the shells: the Hi would represent the diffuse envelope of dense molecular clouds, and this combination would prevent the $\mathrm{H}_{\mathrm{I}}$ to be quickly driven toward the centre during the formation of the shell structures.

The partial ring structure of the $\mathrm{HI}_{\mathrm{I}}$ clouds (Schiminovich et al. 1994) further supports the external origin of the gas in Cen A. Furthermore, recent deep ATCA Hi observations have revealed two more small clouds of $\mathrm{H}$ I located at about 5 and $10 \mathrm{kpc}$ from the centre in the north-east region (see below and Struve et al. 2010a). One cloud is observed in emission and one is seen as $\mathrm{H}_{\mathrm{I}}$ absorption against the radio continuum. This result suggests that more $\mathrm{HI}_{\mathrm{I}}$ could be present. The existence of additional, low mass $\left(<10^{6} \mathrm{M}_{\odot}\right) \mathrm{H}$ i clouds is not surprising. The velocities of the two newly discovered $\mathrm{H}_{\mathrm{I}}$ clouds are in agreement with the spatial velocity distribution (gradient) of the $\mathrm{H}_{\mathrm{I}}$ 'outer ring' structure and their very narrow $\mathrm{H}_{\mathrm{I}}$ profile, suggesting that these clouds are more likely connected with the large scale $\mathrm{H}_{\text {I }}$ structure and are more likely the left over of the interaction/merger discussed above.

It is interesting to note that the $\mathrm{H}_{\mathrm{I}}$ in the outer ring combined with the $\mathrm{H}_{\mathrm{I}}$ in the dust-lane disk (see below) suggests a flat rotation curve $\left(250 \mathrm{~km} \mathrm{~s}^{-1}\right.$ at $15 \mathrm{kpc}$ from the centre). Using this, a value of $M / L \sim 6$ is derived (Schiminovich et al. 1994). This value is consistent with what found for other early-type galaxies from $\mathrm{H}$ i observations (see, e.g., Morganti et al. 1999b). However, a comparison with values derived from the X-ray halo (Forman, Jones \& Tucker 1985) shows that the $M / L$ derived from the X-ray is at least a factor of 4 higher. This discrepancy has not been explained, except by invoking the effect of discrete sources in the X-ray luminosity.

Very interesting is also the distribution of the hot gas. The overall X-ray structure of Cen A was first studied by Feigelson et al. (1981) using Einstein IPC data. These data already revealed how complex the X-ray emission is in this object. Feigelson et al. (1981) identify emission from different structures in addition to the smooth diffuse halo of hot gas. These structures include the radio jet, confirming the early detection of Schreier et al. (1979). The X-ray structures have been further studied with exquisite details thanks to deep Chandra and XMMNewton observations (see Kraft et al. 2009 and references therein). These observations have shown that the X-ray emission from the inner jet and radio lobes display significant differences between the north-east lobe and the south-west lobe. The dominant X-ray emission mechanism from the inner jet is synchrotron radiation emanating from $\sim 30$ knots embedded in diffuse emission. There are significant spatial offsets between the X-ray and radio peaks of the inner jet (Kraft et al. 2002), and proper motions of some radio knots show velocities of $\sim 0.5 c$ (Hardcastle et al. 2003). A shell of shock-heated gas and X-ray synchrotron emitting ultra-relativistic electrons surrounds the SW lobe (Kraft et al. 2003, 2007; Croston et al. 2009). The temperature and density of the gas in the shell is several times that of the ambient medium. Thus, the inflation of the south-west lobe is driving a strong shock into the ISM. Surprisingly, there is no corresponding shell of shock-heated gas around the north-east lobe.

Feigelson et al. (1981) also reported the discovery of an X-ray filament along the south-east edge of the northern middle lobe. This feature was also detected in EXOSAT (Morini et al. 1989), ASCA (Morganti et al. 1999), and in unpublished archival ROSAT PSPC observation. Feigelson et al. (1981) argued that this feature was probably from hot gas, and rejected both synchrotron emission from a population of ultra-relativistic electrons and inverse-Compton scattering of cosmic microwave 

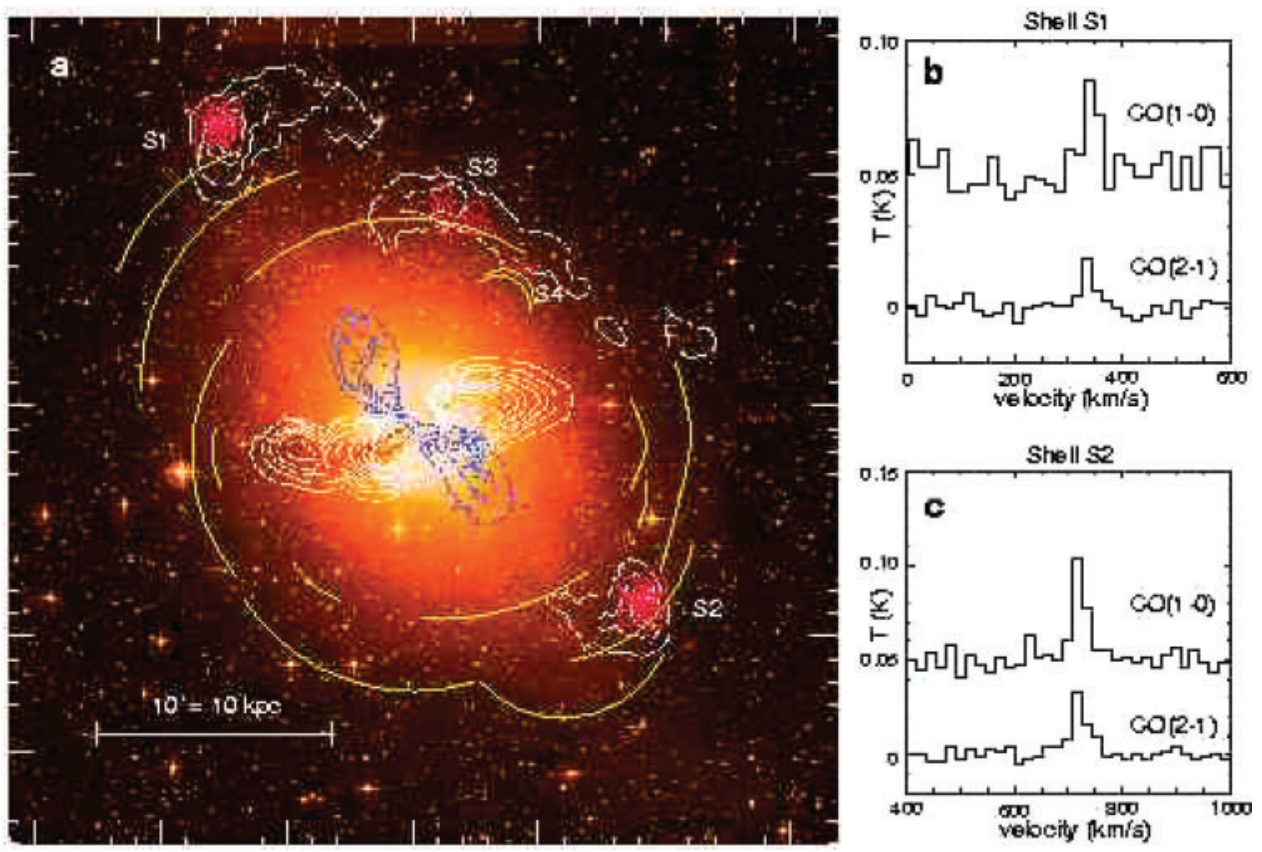

Figure 4 Figure taken from Charmandaris et al. (2000) showing (a) the locations of the various gas components; and $\mathrm{CO}(1-0)$ and $\mathrm{CO}(2-1)$ spectra (b) toward the northern shell and (c) toward the southern shell. (a) shows DSS optical image of Cen A with the (white) contours of the H I gas (Schiminovich et al. 1994). The positions observed in CO by Charmandaris et al. (2000) are marked by circles while the location of the outer stellar shells are underlined by the yellow solid lines. The inner radio lobe is represented by the blue contours (from Clarke, Burns \& Norman 1992).

background (CMB) photons as viable possibilities. A deep image of this region has been obtained by Kraft et al. (2009) using XMM-Newton. They find that the $\mathrm{X}$-ray filament consists of five spatially resolved X-ray knots embedded in a continuous diffuse bridge. Based on energetic arguments, they concluded that it is implausible that these knots have been ionized by the beamed flux from the AGN of Cen A or that they have been shockheated by supersonic inflation of the northern middle lobe. Instead, the most viable scenario for the origin of the $\mathrm{X}$-ray knots appears to be that they are the result of cold gas shock heated by a direct interaction with the jet.

All this clearly illustrate how complex the interaction between the radio jet and the ISM can be, even well outside the nuclear regions.

\section{The Gas along the Dust Lane}

Most of the gas (ionized, atomic neutral and molecular) observed in Cen A is actually located in a disk that follows the dust lane. In this region, the distribution of the dust has been observed by the Spitzer Infrared Array Camera (Leeuw et al. 2002; Quillen et al. 2006), by ISO with ISOCAM and by SCUBA (Mirabel et al. 1999).

The emission from the ionized gas (and in particular $\mathrm{H} \alpha$ emission line) has been studied using the integral field unit TAURUS on the Anglo Australian Observatory (AAO) by Bland, Taylor \& Atherton (1987) and Nicholson, Bland-Hawthorn \& Taylor (1992). Figure 5 illustrates the distribution and kinematics of the ionized gas. Nicholson et al. (1992) presented geometrical, tiltedring models that successfully reproduce not only the appearance of the dust-lane system seen in broad-band optical images but also the main kinematics and morphological features seen in the $\mathrm{H} \alpha$ emission. The structure of the warped disk has been confirmed also by the study of the molecular gas. Eckart et al. (1990) observed Cen A with SEST in the ${ }^{12} \mathrm{CO}(1-0,2-1)$ and ${ }^{13} \mathrm{CO}$ lines with a resolution of about 45 arcsec and 22 arcsec, respectively. Quillen et al. (1992) observed the ${ }^{12} \mathrm{CO}(2-1)$ line using the Caltech Submillimeter Observatory (CSO) in order to obtain a better velocity resolution. The mass estimated for the molecular gas is $4 \times 10^{8} \mathrm{M}_{\odot}$. Figure 6 shows the distribution of the $\mathrm{CO}$ and its kinematics along the dust lane. The models of Nicholson et al. (1992) and Quillen et al. (1992) describe the overall structure observed (from the dust, ionized and molecular gas) as a transient, warped and thin disk composed of a series of inclined connected rings undergoing circular motion. The disk is rapidly rotating with a rotation gradient of about $150 \mathrm{~km} \mathrm{~s}^{-1} \mathrm{kpc}^{-1}$ and highly inclined. Expanding on the work of Nicholson et al. (1992) and Quillen et al. (1992), Quillen, Graham \& Frogel (1993) have suggested a timescale of about $200 \mathrm{Myr}$ since the core of an infalling spiral galaxy reached and merged with Cen A. Because of this encounter, an initially flat disk, misaligned with the galaxy principal symmetry axis, becomes increasingly corrugated as a function of time. An alternative model a polar ring model - to explain the warped structure has been instead proposed by Sparke (1996). This dynamical model shows that the geometry of the disk can be explained as that of a near-polar structure precessing about the symmetry axis of an approximately oblate 

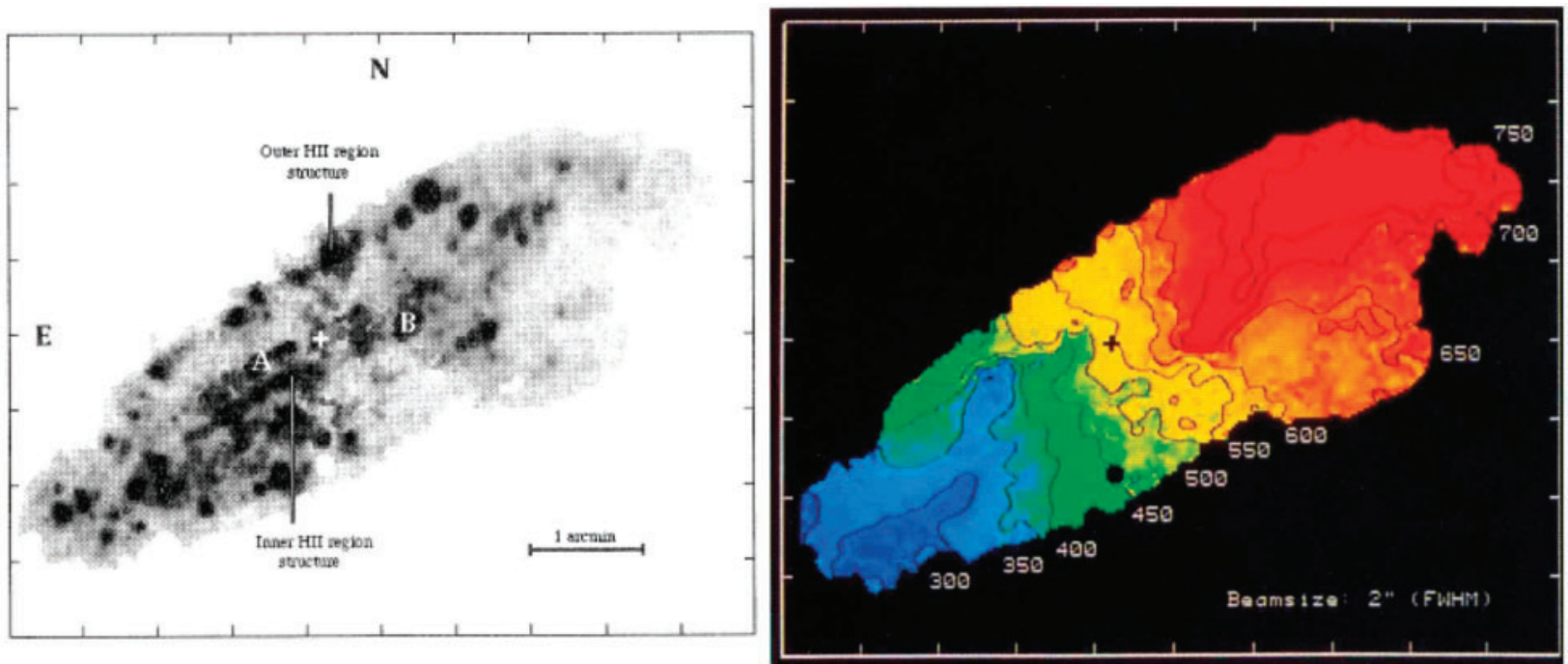

Figure 5 Total intensity and velocity field of the $\mathrm{H} \alpha$ emission line obtained from the TAURUS imaging Fabry-Perot system at the AAT (Bland et al. 1987; Nicholson et al. 1992). The images were kindly provided by Joss Bland-Hawthorn.

(a)

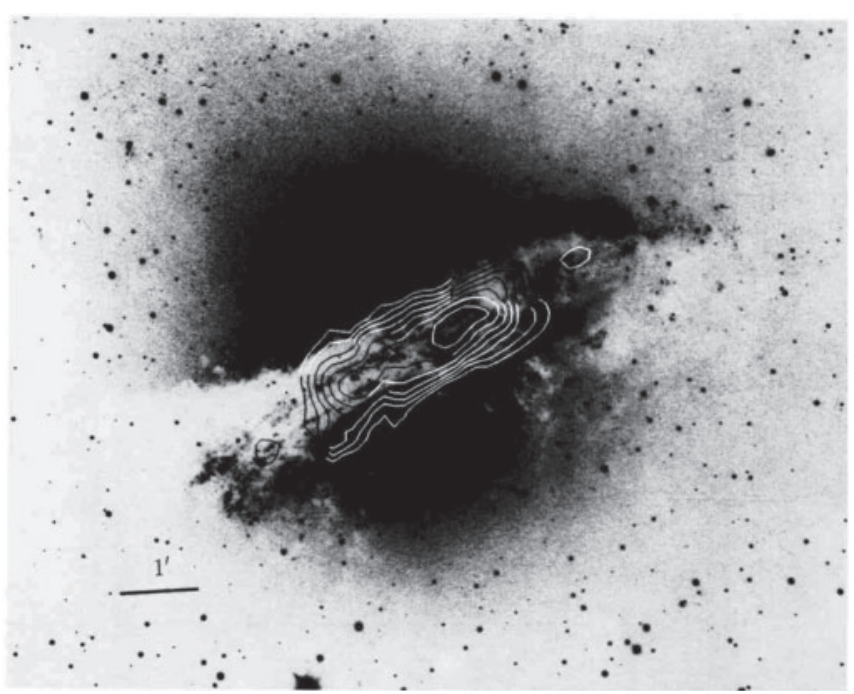

(b)

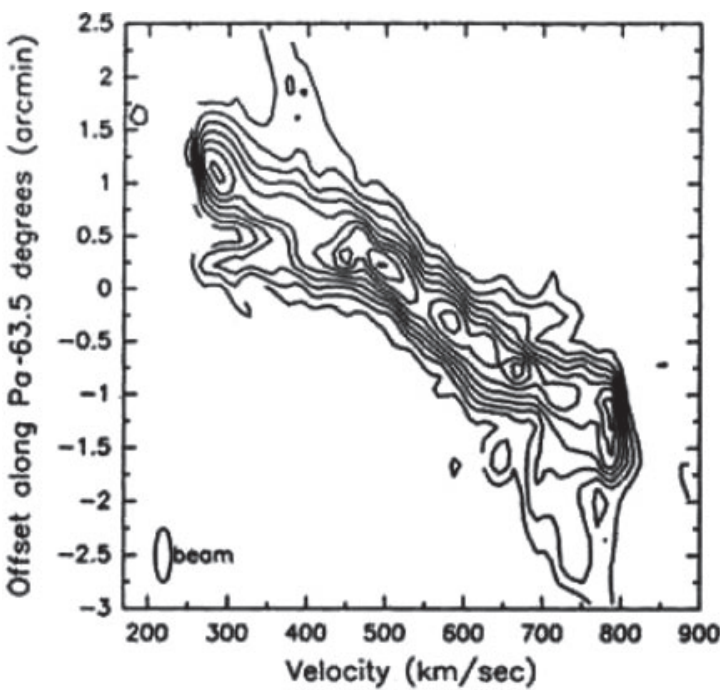

Figure 6 (a) Contour map of the integrated ${ }^{12} \mathrm{CO}(1-0)$ emission in Cen A superimposed to an optical image (taken from Eckart et al. 1990). (b) Position-velocity map of the ${ }^{12} \mathrm{CO}(2-1)$ from Caltech Submillimeter Observatory by Quillen et al. (1992). The plot represents a cut taken along the dust lane. The velocity is given with respect to the systemic velocity.

galaxy potential which is nearly round at the centre (like the stellar body). The outer H I structure described in the previous session (Schiminovich et al. 1994) can be explained as the continuation of the same precessing disk. However, the timescales required by this model to account for the twist in the warp are longer. A summary of the timescales derived from various diagnostics will be given in Section 6.

Another important component of the gas that is present along the dust disk of Cen A is the neutral hydrogen. The distribution and kinematics of the $\mathrm{H}_{\mathrm{I}}$ along the dust lane was first observed using the VLA by van Gorkom et al. (1990). H $\mathrm{I}$ is detected mostly in emission but also in absorption against the southern radio lobe, the core and the beginning of the northern jet. The morphology and kinematics of the $\mathrm{H}_{\mathrm{I}}$ confirmed the warped structure although given the low spatial and kinematic resolution of this data no detailed model was performed. The total mass of the $\mathrm{H}_{\mathrm{I}}$ disk is $3.9 \times 10^{8} \mathrm{M}_{\odot}$.

More recent observations were obtained with the ATCA, achieving relatively high spatial $(\sim 6$ arcsec) and velocity resolution. This has allowed more detailed imaging of the kinematics of the gas (and better separates the emission and absorption components). See Struve et al. (2010a, 2010b) for more details. The quality of the data has allowed a detailed modelling using a tilted ring model of the disk. The kinematics of the $\mathrm{H}_{\mathrm{I}}$ can be described — down to the nuclear scales — as a regularly 
rotating, highly warped structure. Large-scale radial motions do exist, but they are only present at larger radii. This unsettled gas is mainly part of a tail/arm-like structure, possibly wrapped around the radio lobe as it appears both in emission and absorption in the region below the dust lane. The relatively regular velocity of the gas in this structure suggests that it is in the process of settling down into the main disk. The parameters derived from the tilted ring modelling of the $\mathrm{H}$ a are similar but not identical to the ones obtained by Quillen et al. (2006) while they agree very well with the results from the kpc-scale stellar ring discovered by Kainulainen et al. (2009). Furthermore, these parameters give a good description of the Spitzer far-IR data from Quillen et al. (2006). There are no indications of large-scale anomalies in the kinematics all the way down to the central beam $(\sim 100 \mathrm{pc})$. The presence of a large-scale bar structure is also excluded by these data.

It is interesting to note that a large-scale bar structure was suggested from the dust structure imaged with ISO (Mirabel et al. 1999). This structure is clearly ruled out by the more recent Spitzer far-IR data (see Quillen et al. 2006) and not required to explain the $\mathrm{H}$ i data (Struve et al. 2010b). However, the discussion about the possible presence of a bar structure (in the very inner regions) and the connection to fuelling of the AGN is still ongoing (see Section 4). The possible presence of such a structure has relevance for the fuelling of the AGN.

Finally, in addition to the warm and cold gas, arcs of hot gas have been detected in soft X-rays with Chandra (Karovska et al. 2002) extending $\sim 8 \mathrm{kpc}$ in a direction perpendicular to the jet. The thermal gas would have a density between a few $\times 10^{-3}$ and $10^{-2} \mathrm{~cm}^{-3}$. A few hypotheses about the origin of this structure have been put forward. This emission may originate from interaction (and shocks) between infalling gas/tail from merger and the material in the host elliptical galaxy. Alternatively, the origin could be related to the nucleus. The emission would be resulting from an interaction between a powerful outflow (or wind) from the nucleus and the system of stellar shells or the ISM. This giant eruption/outburst would have taken place $\sim 10^{7}$ years ago (by deriving a mean velocity of the ejecta of $\sim 600 \mathrm{~km} \mathrm{~s}^{-1}$; Karovska et al. 2002).

\section{The Sub-kpc Region}

Various studies have pointed out the presence of a gap in the distribution of gas and dust between 10 arcsec and $45 \operatorname{arcsec}$ (from $\sim 0.2$ and $0.8 \mathrm{kpc}$ ). This has been observed in $\mathrm{H} \alpha, \mathrm{CO}$ and, recently, in H I (Nicholson 1992; Quillen et al. 2006; Struve et al. 2010b, respectively). As also discussed in Struve et al. (2010a), ring-like structures are, in fact, seen in a number of early-type galaxies (see Donovan et al. 2009 and references therein). Various possibilities for the origin of this gap have been considered. In particular, the well-studied case of IC 2006 (Schweizer, van Gorkom \& Seitzer 1989) shows that gas

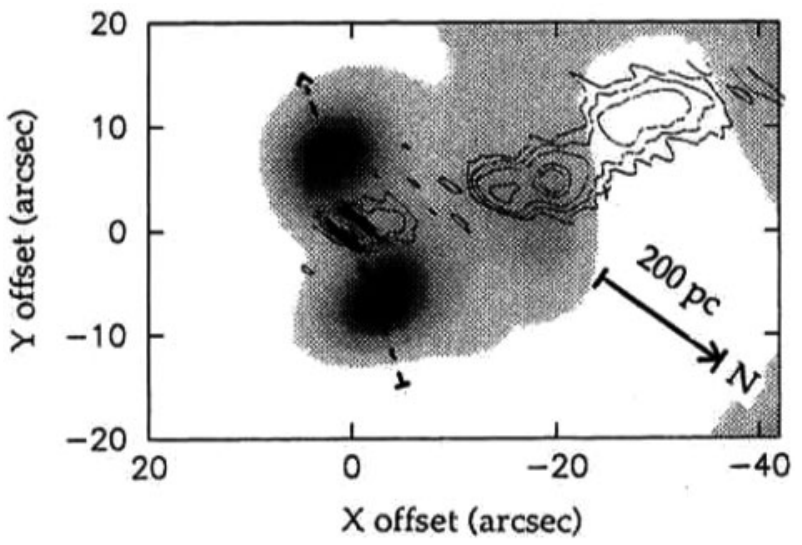

Figure 7 Deconvolved image (grey scale) of the $\mathrm{CO}(2-1)$ obtained by using only the highly red- and blue-shifted gas (from Rydbeck et al. 1993). The contours represent the $1.5 \mathrm{GHz}$ VLA image of the radio jet from Burns, Feigelson \& Schreider (1983).

accretion can also form such structures. In addition to this, effects of inclination or effects related to nuclear energetic processes should also been considered.

On the other hand, gas is present in the nuclear regions of Cen A, i.e. inside the inner $\sim 200 \mathrm{pc}$. The presence of a circumnuclear ring of molecular gas has been inferred for the first time from CO data, thanks to the detection of broad, asymmetric 'high velocity wings' that were interpreted as due to rapidly rotating molecular gas close to the nucleus (Israel et al. 1990). A CO intensity image obtained using only these high velocity wings indeed shows an edge-on ring radius of $\sim 70-120$ pc perpendicular to the radio jet (see Figure 7 and Rydberk et al. 1993). This disk is barely resolved in the ${ }^{12} \mathrm{CO}(3-2)$ data of Liszt (2001) obtained with the JCMT at about 14 arcsec resolution.

This inner molecular disk has been recently observed, with arcsec resolution, in ${ }^{12} \mathrm{CO}(2-1)$ by Espada et al. (2009) using the Submillimeter Array (SMA). These observations spatially resolve the circumnuclear molecular gas in the inner few hundred pc $(400 \mathrm{pc} \times 200 \mathrm{pc})$, revealing that this disk is elongated along a position angle $\sim 155^{\circ}$ and, therefore, perpendicular to the radio/X-ray jet. The south-east and north-west components of the circumnuclear gas are connected to molecular gas found at larger radii, see Figure 8.

Espada et al. (2009) have extened the available models to the inner radii $(r<200 \mathrm{pc})$ in order to reproduce the central parallelogram-shaped structure observed in ${ }^{12} \mathrm{CO}(2-1)$, see Figure 8. Adopting the warped disk model, they confirmed a gap in emission between 200 and $800 \mathrm{pc}$ radii, as mentioned above. However, they also found spatial and kinematical asymmetries in both the circumnuclear and outer gas, suggesting non-complanar and/or non-circular motions. They propose a model combining a weak bar in a warped disk. However, the velocity field derived by their proposed model does not show the large gradient seen in the data in the intersection between the circumnuclear gas and the gas at larger radii, see Figure 8 


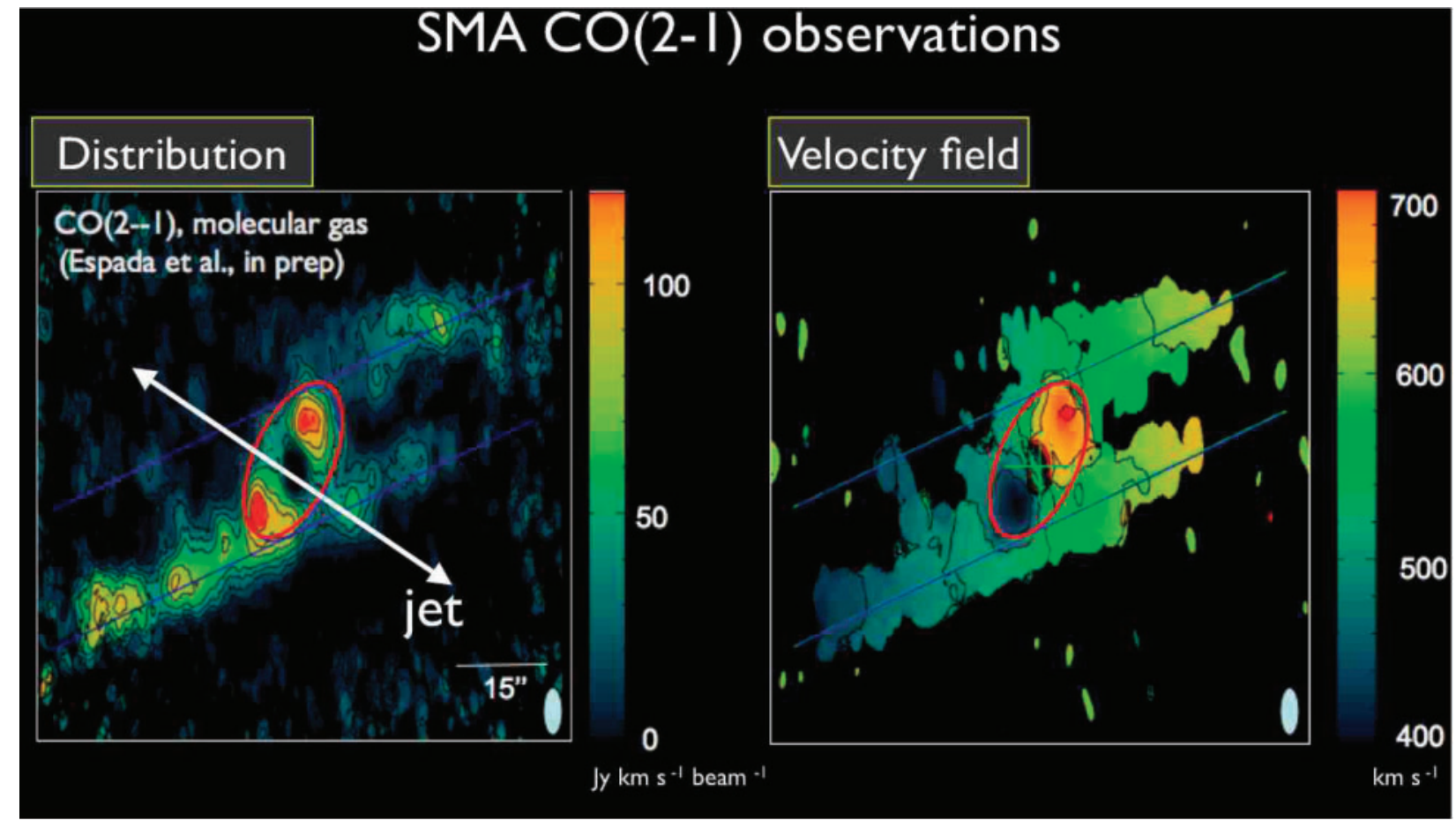

Figure $8{ }^{12} \mathrm{CO}(2-1)$ total intensity (left) and velocity field (right) taken from Espada et al. (2009), Submillimeter Array observations. The colour scale ranges from $400 \mathrm{~km} \mathrm{~s}^{-1}$ up to $700 \mathrm{~km} \mathrm{~s}^{-1}$.

and figure 11 of their paper. More work will need to be done to establish whether a weak bar structure is present in the very centre of Cen A.

The nuclear regions have also been explored with nearIR spectroscopy. Marconi et al. (2001) have presented long slit near-IR spectroscopy of the ionized and molecular gas ([FeII], $\mathrm{Pa} \alpha, \mathrm{Br} \gamma, \mathrm{H}_{2}$ ) in the inner $\sim 200 \mathrm{pc}$. They identify three components. A large scale disk, a 'ring like' system with inner radius 6 arcsec and detected only in $\mathrm{H}_{2}$ and, finally, the likely counterpart of the $100 \mathrm{pc}$ disk seen in CO. However, using these data they could not distinguish between the presence of a bar structure or a warped disk for this inner region of the gas. No optical emission from star formation associated with the radio jet was detected (Marconi et al. 2001). Expanding on these results, higher resolution near-IR observations using AO have allowed a better insight in the very inner regions and will be discussed in the next session.

Toward the central continuum regions of Cen A, many atomic and molecular species have been detected thanks to observations of absorption lines. In particular, Cen A was the first object in which $\mathrm{H}_{\mathrm{I}}$ absorption was detected (Roberts 1970) using single dish observations. Higher resolution observations first suggested that the $\mathrm{H}_{\mathrm{I}}$ absorption against the nuclear region was mostly redshifted (van der Hulst, Golisch \& Haschick 1983; van Gorkom et al. 1989). The conclusion from these observations was that the $\mathrm{H}$ I gas was mainly infalling and likely feeding AGN. However, the deeper ATCA data described above have shown that the situation is more complicated than this. Absorption has been detected against the radio core also at velocities blue-shifted with respect to the systemic velocity (Morganti et al. 2008). The nuclear $\mathrm{H}_{\mathrm{I}}$ absorption appears asymmetric with respect to the systemic velocity (taken as $542 \mathrm{~km} \mathrm{~s}^{-1}$ from van Gorkom et al. 1990), with velocities ranging from $\sim 400 \mathrm{~km} \mathrm{~s}^{-1}$ (about $-140 \mathrm{~km} \mathrm{~s}^{-1}$ blue-shifted compared to systemic) up to $\sim 800 \mathrm{~km} \mathrm{~s}^{-1}$ (i.e. about $+260 \mathrm{~km} \mathrm{~s}^{-1}$ red-shifted relative to systemic), see Figure 9. This absorption profile is, therefore, much broader than reported before. With these new results, the kinematics of the $\mathrm{H}_{\mathrm{I}}$ in the inner regions of Cen A appears very similar to that observed in emission for the molecular circumnuclear disk. In particular, Figure 9 shows how the $\mathrm{H}_{\mathrm{I}}$ absorption compares with the $\mathrm{CO}$ disk observed by Liszt (2001). It is important to note that the modelling of the large-scale $\mathrm{H}_{\mathrm{I}}$ has ruled out that the absorption is due to gas at large distances projected against the centre (Struve et al. 2010b). Thus, these results suggest that the central $\mathrm{H}_{\mathrm{I}}$ absorption does not provide direct evidence of gas infall into the AGN, but instead is (at least partly) due to a cold, circumnuclear disk (Morganti et al. 2008). This also rules out the presence of nuclear fast outflows in Cen A, unlike what has been detected in other radio galaxies. In Cen A, the effects of the interaction between the radio plasma and ISM seem to happen on the larger scale (i.e. inner radio lobes, Croston et al. 2009 or northern middle lobe). However, it is not yet clear what is the illuminating background continuum source at this frequency against which the $\mathrm{HI}_{\mathrm{I}}$ absorption is detected. As shown in Figure 11 (left), the radio core is already not detected at $2.3 \mathrm{GHz}$. Indeed, as shown by Jones et al. (1996) and Tingay \& Murphy (2001), between 

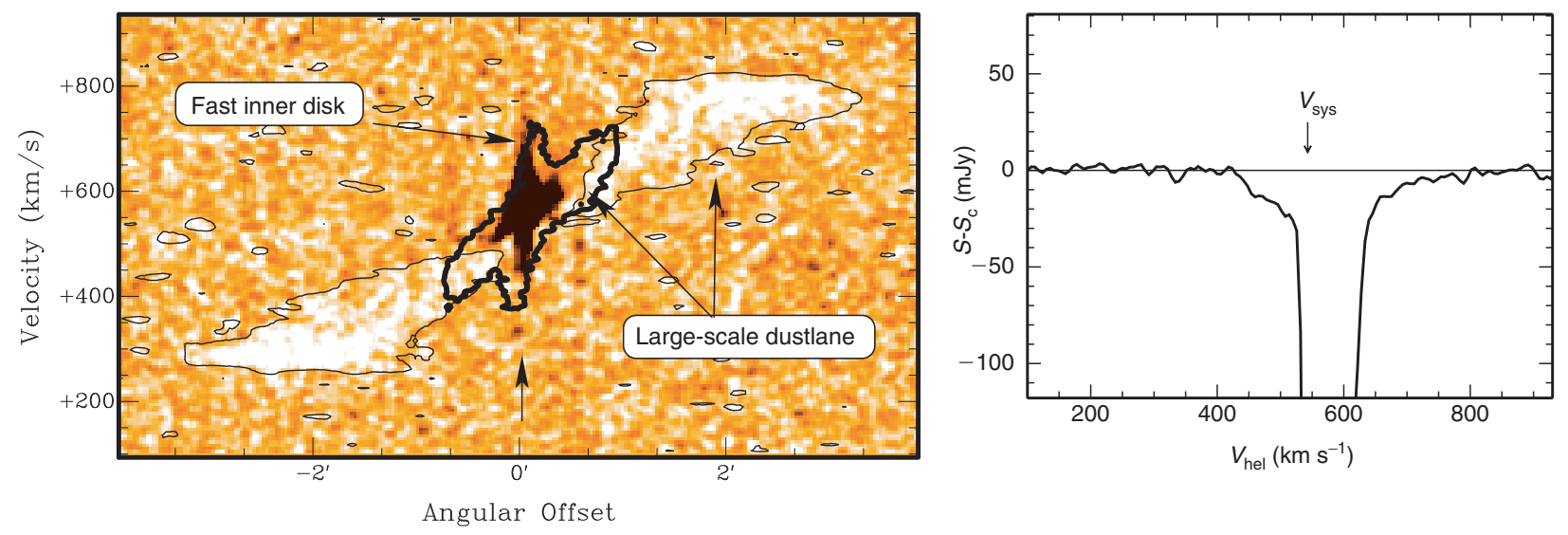

Figure 9 Position-velocity plot (left) of the $\mathrm{H}$ I (grey-scale and thin contours) and, superimposed, the $\mathrm{CO}$ emission (thick contours; from Liszt 2001), taken along position angle $139^{\circ}$. The gray-scale represents the high-resolution $\mathrm{H}$ I data (beam $8 \operatorname{arcsec}$ ) while the thin contour is from the same dataset smoothed to $15 \mathrm{arcsec}$. Note that the $\mathrm{CO}$ observations do not extend beyond a radius of about $1 \mathrm{arcmin}$. The absorption profile (right) against the central radio core (indicated by the arrow in the left panel) showing the blue- and red-shifted wings of the absorption profile.

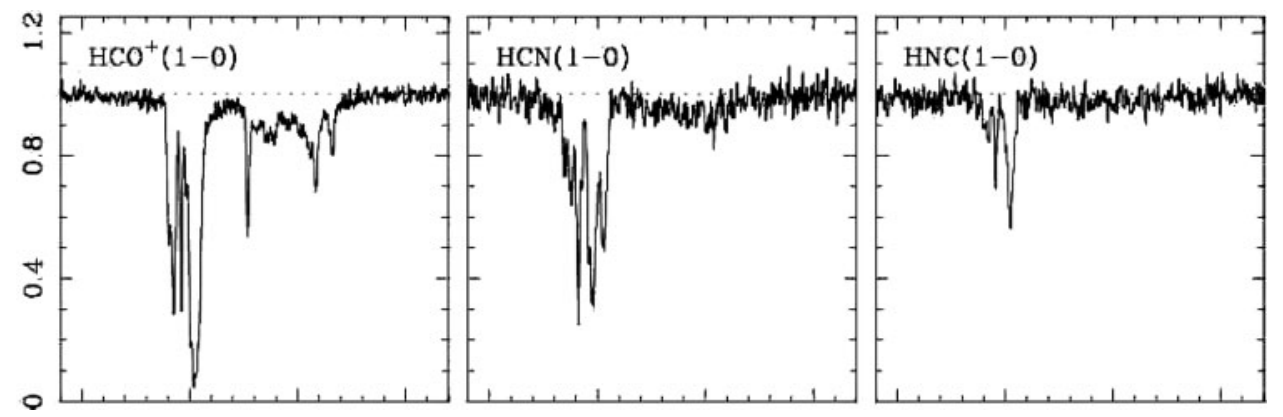

Figure 10 Spectra of the observed transitions detected by Wiklind \& Combes (1997). The spectra have been normalized to a continuum level of unity, see Wiklind \& Combes (1997) for details.

2 and $5 \mathrm{GHz}$ the innermost part of the radio continuum source is affected by free-free absorption that might be caused by circumnuclear ionized gas. The absorption of hard X-rays, indicating a column density of $10^{23}$ atoms $\mathrm{cm}^{-2}$ of absorbing gas in front of the central source (Evans et al. 2004), also suggests the presence of circumnuclear material around the super-massive black hole, as expected from the AGN unification models. Thus, the $\mathrm{H}$ I absorption is occurring either against the counter jet or against a more extended region, e.g., due to thermally emitting ionized gas evaporated from the inner edge of a torus or disk. Deep, high-resolution H I observations will be needed to shed light on this.

Many different molecular lines have been detected against the nucleus in absorption: $\mathrm{H}_{2} \mathrm{CO}, \mathrm{OH}, \mathrm{NH}_{3}, \mathrm{C}_{3} \mathrm{H}_{2}$ (Gardner \& Whiteoak 1976; Seaquist \& Bell 1986, 1990; Israel et al. 1990). For a more complete summary of the detected species see Israel (1998) and Muller \& Dinh-VTrung (2009). As in the case of the $\mathrm{H} \mathrm{I}$, it is still a matter of debate whether these tracers are located in the circumnuclear regions.

$\mathrm{HCO}^{+}$and $\mathrm{CO}$ weak absorption features were detected by Israel et al. (1991) at red-shifted velocities compared to the systemic velocity and explained as gas falling into the nucleus. More observations of the $\mathrm{HCO}^{+}$and other lines were published by Wiklind \& Combes (1997) and Figure 10 shows examples of their molecular absorption profiles. These profiles can be decomposed in a series of deep, very narrow components (with a width of $1-2 \mathrm{~km} \mathrm{~s}^{-1}$ ) located close to the systemic velocity and a broad component. This is, to first order, similar to the $\mathrm{H}_{\mathrm{I}}$ profile although no one-to-one correspondence of the narrow components has been found and the broad component is mostly red-shifted in the molecular gas although a hint of very low optical depth blue-shifted $\mathrm{HCO}^{+}$ absorption wing is also seen (Israel et al. 1992; Wiklind \& Combes 1997). The broad component has been explained by these authors as perhaps originated by a circumnuclear disk. An alternative scenario has been proposed by Eckart, Wild \& Ageorges (1999): the general velocity stucture of the absorption could be explained kinematically with a tilted-ring model and high latitude clouds, not necessarely requiring the presence of molecular gas close to the active nucleus. However, the thickness would not be enough to explain, e.g., the range of velocities detected in $\mathrm{H}$.

The results from VLBI observations of the $\mathrm{OH}$ transitions (van Langevelde, Pihlstróm \& Beasley 2004), as 

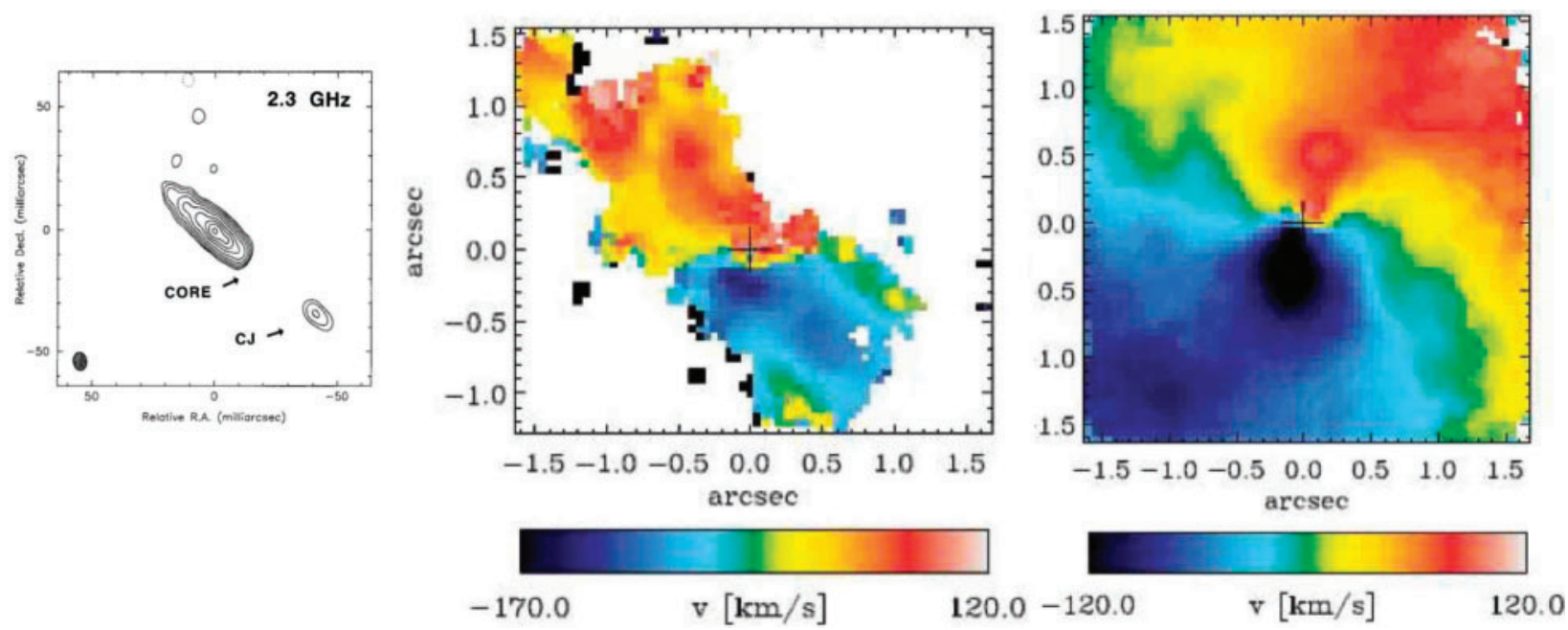

Figure 11 Panel showing (left) the 2.3 GHz VLBI jet with the location of the (free-free absorbed) core (Jones et al. 1996); (middle) the velocity map of the [SivI] line emission with the velocity scale given relative to the systemic velocity of the galaxy (assumed $532 \mathrm{~km} \mathrm{~s}^{-1}$ ) from Neumayer et al. (2007); (right) same for as middle pannel but for $\mathrm{H}_{2}$ (Neumayer et al. 2007).

well as the excitation modelling, appear to be also consistent with the absorption occurring on $200-2000 \mathrm{pc}$ from the centre of Cen A. On the other hand, the formaldehyde absorption detected on the VLBI scale seems to have a distinctly different distribution, possibly restricted to small, high-density clouds in the circumnuclear disk (van Langevelde et al. 2004).

In general, it is clear that the physical conditions of the absorbing molecular gas are still not completely understood. For example, recent observations of $\mathrm{HCN}^{+}$and HCN by Muller \& Dinh-V-Trung (2009) found no evidence for molecular gas density higher than a few $\times 10^{4} \mathrm{~cm}^{-3}$ (unlike previous claims), thus suggesting that either the line-of-sight to the radio continuum does not intercept the circumnuclear disk or the density of the absorbing gas in the circumnuclear disk is lower than $10^{4} \mathrm{~cm}^{-3}$.

\section{The Very Inner Region $(<30 \mathrm{pc})$}

Ionized and molecular gas in the inner $\sim 30 \mathrm{pc}$ have been studied, using integral field spectroscopy, by Krajnović, Sharp \& Thatte (2007) and Neumayer et al. (2007). The latter made use of SINFONI AO-assisted at the VLT to achieve the highest possible spatial resolution. Neumayer et al. (2007) found that for higher excitation lines [SivI] and [FeII] the velocity pattern is increasingly dominated by a non-rotating component, elongated along radio jet. Interestingly, these non-rotational motions were detected in the direction along the radio jet, with red-shifted velocities (compared to the systemic) seen on the main-jet side and blue-shifted on the counter-jet side. These motions (stronger in [Sivi]) can be explained as backflow of gas accelerated by the plasma jet.

On the other hand, the velocity of the $\mathrm{H}_{2}$ line is dominated by rotation, although with a quite high dispersion $\left(\sigma \sim 400 \mathrm{~km} \mathrm{~s}^{-1}\right.$ for the $\mathrm{H}_{2}$ line and higher for the other lines). The reason for this high value of the velocity dispersion is not clear but partially resolved rotation or local turbulence have been considered. All in all, the $\mathrm{H}_{2}$ data in Neumayer et al. (2007) appear to be fully described by a tilted-ring model and gas moving in circular orbits. On such a small scale, the origin of the structure of the nuclear disk could be due to self-induced warping of the accretion disk.

Although the overall shape of the $\mathrm{H}_{2}$ velocity field appears to be point-symmetric, Neumayer et al. (2007) point out some asymmetries of the peak velocities in the field. These asymmetries may be related to the fuelling process of the nuclear disk suggested by the morphology and kinematics of the high-ionization lines. Interestingly, the range of velocities covered by the $\mathrm{H}_{\mathrm{I}}$ absorption against the nuclear regions (Morganti et al. 2008) is similar to the range covered by the near-IR lines on the blue-shifted side, while they are larger on the red-shifted side (extending to $\sim 800 \mathrm{~km} \mathrm{~s}^{-1}$ while the $\mathrm{H}_{2}$ and [SivI] cover only up to $\sim 650 \mathrm{~km} \mathrm{~s}^{-1}$ ).

One of the results from these IFU studies is also the determination of the BH mass in Cen A. From the Neumayer et al. (2007) tilted-ring model of the gas, a BH mass $4.5 \times 10^{7} \mathrm{M}_{\odot}$ has been derived (for an inclination $i=34^{\circ}$ ). This is lower than found in previous studies. The difference (and improvement) is attributed to the fact that lines with more regular rotation (i.e., less affected by the radio jet) have been used. Interestingly, a similar BH mass has been obtained from the stellar kinematics (Cappellari et al. 2009). The new estimated value of the BH fits nicely in the $\mathrm{M}_{\mathrm{BH}^{-}}-\sigma$ correlation (see figure 17 in Neumayer et al. 2007).

Finally, it is worth mentioning that, using X-ray data, Evans et al. (2004) estimated the accretion rate (Bondi accretion) of gravitationally captured hot gas into the central BH to be $\sim 6.4 \times 10^{-4} \mathrm{M} \mathrm{yr}^{-1}$ and a corresponding efficiency of $\sim 0.2 \%$. These values (although derived using old values of the $\mathrm{BH}$ mass and, therefore, requiring an update) suggest that Cen A has intermediate characteristics. The value of the accretion rate is higher than in 
galaxies with radiatively inefficient fuelling of the AGN and smaller than for a canonical efficient accretion system. Nevertheless, as mentioned above, the high column density as well as the presence of $\mathrm{Fe} \mathrm{Ka}$ line derived from the X-ray observations (Evans et al. 2004) support the idea that large quantities of cold gas located in a molecular torus at $\sim 1 \mathrm{pc}$ from the $\mathrm{BH}$ are present in Cen $\mathrm{A}$.

\section{Timescales and Concluding Remarks}

Gas structures have been observed in Cen A ranging from many tens of kpc to sub-pc scales. The external origin of the gas via accretion of a small, gas-rich galaxy is supported by some of the observed characteristics, like the misalignment of the rotation axis between stars and gas and the presence of shells. Although this merger may be the origin of the warped structure of the disk, the gas in the disk appears now well settled in a regular structure (warped thin disk and on circular orbits) down to the nuclear regions.

Given the variety of diagnostics that can be observed in Cen A, it is interesting to put together some of the timescales involved in producing the observed structures, i.e.

- timescale to create shells from merger is between 2 and $6 \times 10^{8}$ yr (Quillen et al. 2006);

- timescale (to form a warp) from the precessing model is $\sim 7 \times 10^{8}$ yr (Sparke 1996);

- timescale to create the warp is $\sim 2 \times 10^{8} \mathrm{yr}$ (e.g., from CO and Spitzer data; Quillen et al. 1992, 2006);

- timescale obtained from the structure and kinematics (and the modelling) of the $\mathrm{H}$ i disk is $1.6-3.2 \times 10^{8} \mathrm{yr}$ since the merging event (van Gorkom et al. 1990; Struve et al. 2010a);

- ages of stars associated with the young blue tidal stream are 300 Myr (Peng et al. 2002);

- ages of young stars and star clusters in the centre are between $10^{6}$ to a few times $10^{7}$ yr (Dufour et al. 1979; Möellenhoff 1981; Minniti et al. 2004);

- much longer ages - of the order of a few Gyr - are derived from planetary nebulae and globular clusters (Peng, Ford \& Freeman 2004a, 2004b);

- age of the large radio structure (e.g., NML) is estimated to be a few $\times 10^{7} \mathrm{yr}$ from the still ongoing particle injection (Hardcastle et al. 2008) and up to $10^{8} \mathrm{yr}$ if the northern middle radio lobe is described as a rising buoyant bubble structure (Saxton, Sutherland \& Bicknell 2001); and

- age of the inner radio lobe is a few $\times 10^{6} \mathrm{yr}$ from X-ray emission (Croston et al. 2009).

Thus, the timescales derived for the formation of the warped disk structure are much longer than the timescale of the inner radio lobes, and more similar to the timescales derived for the middle radio lobe (see Section 6). This raises the question of whether the merger/accretion and AGN activity are actually at all related.

The fuelling mechanism of the nuclear activity is also not completely clear. However, the very high resolution of near-IR observations points to evidence for some infalling gas that may do the work. There seems to be no obvious evidence for outflows in the nuclear regions while there is evidence on the larger $(\mathrm{kpc})$ scale of interaction between the radio jet and the ISM. These lobes are only $6 \mathrm{kpc}$ in radius, so this may represent what commonly happens in young or restarted radio sources when they reach the kpc scales. This would, therefore, confirm how the early stages of radio-galaxy evolution potentially represent an important galaxy feedback process.

\section{References}

Baum, S., Heckman, T. M., Bridle, A., van Breugel, W. J. M. \& Miley, G. K., 1988, ApJS, 68, 643

Bender, R., Burstein, D. \& Faber, S. M., 1992, ApJ, 399, 462

Blanco, V. M., Graham, J. A., Lasker, B. M. \& Osmer, P., 1975, ApJ, 198, L63

Bland, J., Taylor, K. \& Atherton, P. D., 1987, MNRAS, 228, 595

Bournaud, F., Jog, C. J. \& Combes, F., 2005, A\&A, 437, 69

Burns, J. O., Feigelson, E. D. \& Schreider, E. J., 1992, ApJ, 273, 128

Cappellari, M. et al., 2008, MNRAS, 379, 445

Cappellari, M. et al., 2009, MNRAS, 394, 660

Charmandaris, V., Combes, F. \& van der Hulst, J. M., 2000, A\&A, 356, L1

Combes, F., Young, L. M. \& Bureau, M., 2007, MNRAS, 377, 1795

Clarke, D. A., Burns, J. O. \& Norman, M. L., 1992, ApJ, 395, 444

Croston, J. H. et al., 2009, MNRAS, 395, 1999

Dekel, A. et al., 2009, Nature, 457, 451

Donovan, J. et al., 2009, AJ, 137, 5037

Dufour, R. J. \& van den Bergh, S., 1978, ApJ, 226, L73

Dufour, R. J. et al., 1979, AJ, 84, 284

Eckart, A. et al., 1990, ApJ, 363, 451

Eckart, A., Wild, W. \& Ageorges, N., 1999, ApJ, 516, 769

Espada, D. et al., 2009, ApJ, 695, 116

Evans, D. A. et al., 2004, ApJ, 612, 786

Faber, S. M. et al., 1997, AJ, 114, 177

Feigelson, E. D., Schreier, E. J., Delvaille, J. P., Giacconi, R., Grindlay, J. E. \& Lightman, A. P., 1981, ApJ, 251, 31

Forman, W., Jones, C. \& Tucker, W., 1985, ApJ, 293, 102

Fosbury, R. A. E. et al., 1998, MNRAS, 296, 701

Gardner, F. F. \& Whiteoak, J. B., 1976, MNRAS, 175, 9

Graham, J. A., 1998, ApJ, 502, 245

Hardcastle, M. J. et al., 2003, ApJ, 593, 169

Hardcastle, M. J., Cheung, C. C., Feain, I. J. \& Stawarz, L., 2009, MNRAS, 393, 1041

Harris, G. L. H. et al., 2010, PASA, 27, 457

Hopkins, P. F., Lauer, T. R., Cox, T. J., Hernquist, L. \& Kormenndy, J., 2009, ApJS, 181, 486

Israel, F., 1992, A\&A, 265, 487

Israel, F., 1998, A\&A Rev, 8, 237

Israel, F. P., van Dishhoeck, E. F., Baas, F., Koorneef, J., Black J. H. \& de Graauw, S. S., 1990, AJ, 227, 342

Israel, F. P., van Dishhoeck, E. F., Baas, F., de Graauw, S. S. \& Phillips, T. G., 1991, A\&A, 245, L13

Józsa, J. et al., 2009, A\&A, 500, L33

Jones, D. L. et al., 1996, ApJ, 466, L63

Kainulainen, J. T. et al., 2009, A\&A, 502, L5

Karovska, M. et al., 2002, ApJ, 577, 114

Keres, D. et al., 2005, MNRAS, 363, 2

Kormendy, J. \& Bender, R., 1996, ApJ, 464, L119

Kraft, R. P. et al., 2002, ApJ, 569, 54

Kraft, R. P. et al., 2003, ApJ, 592, 129

Kraft, R. P. et al., 2007, ApJ, 665, 1129

Kraft, R. P. et al., 2009, ApJ, 698, 2036

Krajnović, D., Sharp, R. \& Thatte, N., 2007, MNRAS, 374, 385 
Leeuw, L. L., Hawarden, T. G., Matthews, H. E., Robson, E. I. \& Eckart, A., 2002, ApJ, 561, 131

Liszt, H. S., 2001, AJ, 371, 865

Marconi, A. et al., 2000, ApJ, 528, 276

Marconi, A. et al., 2001, ApJ, 549, 915

Mathews, W. G. \& Brigenti, F., 2003, ARA\&A, 41, 191

Minniti, D., Rejkuba, M., Funes, J. G. \& Kennicutt, R. C., 2004, ApJ, 612,215

Mirabel, I. F. et al., 1999, A\&A, 341, 667

Möllenhoff, C., 1981, A\&A, 99, 341

Morganti, R., Robinson, A., Fosbury, R. A. E., di Serego Alighieri, S., Tadhunter, C. N. \& Malin, D., 1991, MNRAS, 249, 91

Morganti, R., Fosbury, R. A. E., Hook, R. N., Robinson, A. \& Tsvetanov, Z., 1992, MNRAS, 256, 1

Morganti, R., Killeen, N. E. B., Ekers, R. D. \& Oosterloo, T. A., 1999a, MNRAS, 307, 750

Morganti, R., Oosterloo, T., Sadler, E. M. \& Vergani, D., 1999b, in Star Formation in Early Type Galaxies, ASP Conf. Ser. 163, Eds. Carral, P. \& Cepa, J., 84

Morganti, R. et al., 2006, MNRAS, 371, 157

Morganti, R., Oosterloo, T., Struve, C. \& Saripalli, L., 2008, A\&A, 485, L5

Morini, M., Anselmo, F. \& Molteni, D., 1989, ApJ, 347, 750

Mould, J. R. et al., 2000, ApJ, 536, 266

Muller, S. \& Dinh-V-Trung, 2009, ApJ, 696, 176

Naab, T. \& Burkert, A., 2003, ApJ, 597, 893

Neff, S. G., Schiminovich, D. \& Martin, C. D., GALEX Science Team, 2003, AAS, 103, 9607

Neumayer, N. et al., 2007, ApJ, 671, 1329

Nicholson, R. A., Bland-Hawthorn, J. \& Taylor, K., 1992, ApJ, 387,503

Oosterloo, T. A. \& Morganti, R., 2005, AJ, 429, 469

Peng, E. W., Ford, H. C., Freeman, K. C. \& White, R. L., 2002, AJ, 124, 3144

Peng, E. W., Ford, H. C. \& Freeman, K. C., 2004a, ApJ, 602, 685

Peng, E. W., Ford, H. C. \& Freeman, K. C., 2004b, ApJ, 602, 705

Quillen, A. C., de Zeeuw, P. T., Phinney, E. S. \& Phillips, T. G., 1992, ApJ, 391, 121
Quillen, A. C. et al., 2006, ApJ, 645, 1092

Quillen, A. C., Graham, J. R. \& Frogel, J. A., 1993, ApJ, 412, 550

Rejkuba, M., Minniti, D., Courbin, F. \& Silva, D. R., 2002, ApJ, 564,688

Roberts, M. S., 1970, ApJ, 161, L9

Rydbeck, G. et al., 1993, AJ, 270, L13

Tingay, S. J., Jauncey, D. L., Reynolds, J. E. et al., 1998, ApJ, 115, 960

Sarma, A. P., Troland, T. H. \& Rupen, M. P., 2002, ApJ, 564, 696

Sarzi, M. et al., 2006, MNRAS, 366, 1151

Saxton, C. J., Sutherland, R. S. \& Bicknell, G. V., 2001, ApJ, 563,103

Schiminovich, D., van Gorkom, J. H., van der Hulst, J. M. \& Kasow, S., 1994, ApJ, 423, L101

Schreier, E. J. et al., 1979, ApJ, 234, L39

Seaquist, E. R. \& Bell, M. B., 1986, ApJ, 303, L67

Seaquist, E. R. \& Bell, M. B., 1990, ApJ, 364, 94

Sparke, L., 1996, ApJ, 473, 810

Struve, C., Morganti, R., Oosterloo, T. \& Emonts, B., 2010a, PASA 27,390

Struve, C., Oosterloo, T., Morganti, R. \& Saripalli, L., 2010b, A\&A, in press

Sutherland, R. S., Bicknell, G. V. \& Dopita, M. A., 1993, ApJ, 414, 510

Schweizer, F., van Gorkom, J. H. \& Seitzer, P., 1989, ApJ, 338, 770

Tingay, S. J. \& Murphy, D. W., 2001, ApJ, 546, 210

van der Hulst, J. M., Golisch, W. F. \& Haschick, A. D., 1983, ApJL, 264, 37

van Gorkom, J. H., van der Hulst, J. M., Haschick, A. D. \& Tubbs, A. D., 1989, AJ, 97, 708

van Langevelde, H. J., Pihlstróm, Y. \& Beasley, A., 2004, in Dense Molecular Gas around Protostars and in Galactic Nuclei, Eds. Hagiwara, Y., Baan, W. A. \& van Langevelde, H. J., ApSS, 295, 249

Wiklind, T. \& Combes, F., 1997, A\&A, 324, 51 